\title{
Spatial Models of Bistability in Biological Collectives
}

\author{
Derek A. Paley, Naomi Ehrich Leonard, Rodolphe Sepulchre and Iain D. Couzin
}

\begin{abstract}
We explore collective behavior in biological systems using a cooperative control framework. In particular, we study a hysteresis phenomenon in which a collective switches from circular to parallel motion under slow variation of the neighborhood size in which individuals tend to align with one another. In the case that the neighborhood radius is less than the circular motion radius, both circular and parallel motion can occur. We provide Lyapunov-based analysis of bistability of circular and parallel motion in a closed-loop system of selfpropelled particles with coupled-oscillator dynamics.
\end{abstract}

\section{INTRODUCTION}

In this paper, we use a cooperative control approach to model collective motion in biology. Collective motion appears in natural systems ranging from molds [1] to locusts [2] to fish [3]. All of these systems exhibit collective motion that ranges from relatively disordered (swarm motion) to highly ordered (parallel motion). Another highly ordered type of motion observed in biological collectives is circular motion [1]-[3], characterized by collective rotation about a fixed point. Numerical investigations [4] of a repulsion/orientation/attraction (ROA) description of collective behavior [4], [5] indicates that changes in model parameters generate transitions between swarm, circular, and parallel motion. In fact, slow variation of a single parameter related to interaction between individuals is observed to generate hysteresis in the transition between these motions.

We infer from the presence of hysteresis in the ROA behavior that there exists a parameter range in which both circular and parallel motion are stable, in a qualitative sense. We say that the ROA behavior is bistable with respect to circular and parallel motion. We seek to prove the existence of such a parameter range in a related model by showing that both circular and parallel motion are stable, in a Lyapunov sense. An earlier version of this work appeared in [6], where we studied a bistable model restricted to two individuals; in this work, that restriction is lifted. Techniques that support our results are drawn from our related work on collective motion in engineered systems [7], [8] as well as from stability theory, graph theory [9], and studies of consensus in timevarying systems [10]. This paper reveals that an alternate

This work was partially supported by an NSF GRF, ONR grants N0001402-1-0826, N00014-02-1-0861 and N00014-04-1-0534, and the Belgian Program on Interuniversity Attraction Poles, initiated by the Belgian Federal Science Policy Office. The scientific responsibility rests with its authors.

D. Paley (dpaley@umd.edu) is an assistant professor of aerospace engineering at the University of Maryland, College Park, MD 20742. N. Leonard (naomi@princeton.edu) is a professor of mechanical and aerospace engineering at Princeton University, Princeton, NJ 08544. R. Sepulchre (r.sepulchre@ulg.ac.be) is a professor of electrical engineering and computer science at the University of Liege, B-4000 Liege Sart-Tilman, Belgium. I. Couzin (icouzin@ @rinceton.edu) is an assistant professor of ecology and evolutionary biology at Princeton University, Princeton, NJ 08544. version of the circular formation control that appeared in [11] also stabilizes parallel formations.

The paper is organized as follows. In Section II, we describe a collective behavior model including the ROA behavior and, in Section III, we present an idealized version of the model that is mathematically tractable. Our approach to developing a provably bistable behavior depends on whether the interaction between individuals in the model is undirected or directed; we describe the approach for undirected interaction in Section IV. The corresponding analysis for directed interaction is omitted, and will be presented elsewhere.

\section{COLLECTIVE BEHAVIOR MODEL}

Each individual in the collective behavior model is represented by a particle (point mass). Let $N$ denote the number of particles. Let $\mathcal{I} \triangleq\left(O, \hat{e}_{x}, \hat{e}_{y}, \hat{e}_{z}\right)$ denote an inertial reference frame with origin $O$ and orthonormal unit vectors $\hat{e}_{x}, \hat{e}_{y}$, and $\hat{e}_{z}$. Let $r_{k}(t)$ denote the position of particle $k \in \mathcal{N} \triangleq$ $\{1, \ldots, N\}$ and $\dot{r}_{k}(t)$ denote the inertial velocity.

Let $\mathcal{B}_{k}$ be a moving reference frame with origin $r_{k}(t)$ and unit vectors $\hat{b}_{k}(t), \hat{a}_{k}(t)$ and $\hat{b}_{k}(t) \times \hat{a}_{k}(t)$ (for example, $\mathcal{B}_{k}$ could be a Fermi-Walker frame [12], although this choice is not critical to the development). Frame $\mathcal{B}_{k}$ is oriented such that $\hat{b}_{k}(t)$ is always parallel to the direction of motion of particle $k$. Let $s_{k}$ denote the speed of particle $k$ and assume that $s_{k}>0$ is constant. The velocity $\dot{r}_{k}(t)$ expressed as a vector component in frame $\mathcal{B}_{k}$ is $\dot{r}_{k}(t)=s_{k} \hat{b}_{k}(t)$.

The trajectory of each particle is determined by a discretetime update rule. Let $\Delta t$ denote the discrete time step. We have $r_{k}(t+\Delta t)=r_{k}(t)+s_{k} \hat{b}_{k}(t+\Delta t)$, where $\hat{b}_{k}(t+\Delta t)$ is a unit vector that represents the direction of motion of particle $k$ at time $t+\Delta t$. The direction of motion of particle $k$ depends on the position and direction of motion of a subset of all of the particles. Let $\mathbf{r}(t) \triangleq\left(r_{1}(t), \ldots, r_{N}(t)\right)^{T}$ denote the $N \times 1$ matrix of particle positions and let $\hat{\mathbf{b}}(t) \triangleq$ $\left(\hat{b}_{1}(t), \ldots, \hat{b}_{N}(t)\right)^{T}$ denote the $N \times 1$ matrix of particle direction unit vectors. The direction of motion $\hat{b}_{k}(t+\Delta t)$ is a function-called a steering behavior-of the entries of $\mathbf{r}(t)$ and $\hat{\mathbf{b}}(t)$ and the time step $\Delta t$.

The subset of particles that interact with particle $k$ and the nature of that interaction depends on the perceptual geometry of particle $k$. Consider $M$ non-overlapping perceptual zones associated to each particle $k$ that are fixed with respect to frame $\mathcal{B}_{k}$. Let $\Gamma_{k}^{(n)}$ denote perceptual zone $n \in\{1, \ldots, M\}$ of particle $k$. If $r_{j}(t) \in \Gamma_{k}^{(n)}(t)$, then particle $k$ perceives particle $j$ and may respond to it; the response of particle $k$ to the presence of particle $j$ depends on $n$. Let $\mathcal{N}_{k}^{(n)}(t)$ denote the set of indices of all of the particles contained in zone $\Gamma_{k}^{(n)}$ at time $t$. For each $n$, the collection of perceptual 
relationships between all of the particles with respect to zone $\Gamma_{k}^{(n)}$ is called an interaction network, of which there are $M$. Let $b_{k}^{(n)}(t+\Delta t)$ denote the desired direction of motion of particle $k$ with respect to the set $\mathcal{N}_{k}^{(n)}(t)$. We call $b_{k}^{(n)}(t+\Delta t)$, which is not necessarily a unit vector, a behavior rule. A steering behavior $\hat{b}_{k}(t+\Delta t)$, such as the ROA behavior described below, satisfies $\hat{b}_{k}(t+\Delta t) \in$ $\operatorname{span}\left\{b_{k}^{(1)}(t+\Delta t), \ldots, b_{k}^{(M)}(t+\Delta t)\right\}$.

We describe a spherical perceptual zone geometry with $M=3$ and the associated behavior rules as defined in [4]. Consider a single right cone with the cone axis lying along $-\hat{b}_{k}(t)$ axis and apex at $r_{k}(t)$. Let $2 \beta_{k}^{(n)}$ denote the opening angle of the cone. Let $\Gamma_{k}^{(n)} \triangleq\left(\beta_{k}^{(n)}, \rho_{k}^{n}\right)$ denote the perceptual zone that is contained in the spherical annulus centered at $r_{k}(t)$ with width $\Delta \rho_{k}^{(n)} \triangleq \rho_{k}^{(n)}-\rho_{k}^{(n-1)}$, where $\rho_{k}^{(n)}$ is the radius of zone $n$ and $\rho_{k}^{(0)}=0$. The portion of the annulus inside the cone, called a blind spot, is not contained in $\Gamma_{k}^{(n)}$. If $\beta_{k}^{(n)}=0$ for all $n$, then particle $k$ has omnidirectional perception. Otherwise, particle $k$ has directional perception.

Three fundamental behavior rules associated with the spherical perceptual zone geometry are repulsion, orientation, and attraction [4], [5]. The first behavior rule, repulsion, generates a desired direction of motion away from the centroid of the particles contained in the innermost perceptual zone $\Gamma_{k}^{(1)}$, which we call the zone of repulsion. Let $r_{k j}(t) \triangleq$ $r_{k}(t)-r_{j}(t)$ denote the position of particle $j$ relative to the position of particle $k$ and let $\hat{r}_{k j}(t) \triangleq r_{k j}(t) /\left|r_{k j}(t)\right|$. The repulsion behavior rule depends on the relative positions $r_{k j}(t)$ for all $j \in \mathcal{N}_{k}^{(1)}(t)$ in the following way: $b_{k}^{(1)}(t+$ $\Delta t)=\sum_{j \in \mathcal{N}_{k}^{(1)}(t)} \hat{r}_{k j}(t)$. The second behavior rule, orientation, generates a desired direction of motion in the average direction of motion of all of the particles in the middle zone $\Gamma_{k}^{(2)}$, which we call the zone of orientation. The orientation behavior rule is $b_{k}^{(2)}(t+\Delta t)=\hat{b}_{k}(t)+\sum_{j \in \mathcal{N}_{k}^{(2)}(t)} \hat{b}_{j}(t)$. The third behavior rule, attraction, generates a desired direction of motion that points toward the centroid of the particles contained in the outermost perceptual zone $\Gamma_{k}^{(3)}$, which we call the zone of attraction. The attraction behavior is $b_{k}^{(3)}(t+\Delta t)=-\sum_{j \in \mathcal{N}_{k}^{(3)}(t)} \hat{r}_{k j}(t)$.

We describe a steering behavior from [4] based on a spherical perceptual zone geometry and the corresponding repulsion, orientation, and attraction behavior rules. In this steering behavior, which we call the ROA behavior, all of the particles move at the same constant speed $s_{0}$. The steering behavior prioritizes the repulsion behavior rule by making it the only active rule at time $t$ if the set $\mathcal{N}_{k}^{(1)}(t)$ is not empty. The steering behavior also limits the maximum absolute rate $\omega_{0}$ at which the particle direction of motion can change over time. Assume $\omega_{0}$ satisfies $0<\omega_{0} \Delta t \leq \pi / 2$. Let

$$
f_{k}(t+\Delta t) \triangleq\left\{\begin{array}{cc}
b_{k}(t), & N_{k}^{(n)}(t)=\emptyset \forall n, \\
b_{k}^{(1)}(t+\Delta t), & \left|\mathcal{N}_{k}^{(1)}(t)\right|>0, \\
\frac{1}{2} \sum_{n=2}^{3} b_{k}^{(n)}(t+\Delta t), & \text { otherwise, }
\end{array}\right.
$$

and $\hat{f}_{k}(t+\Delta t) \triangleq f_{k}(t+\Delta t) /\left|f_{k}(t+\Delta t)\right|$. Let $R_{k}(t, \phi)$ denote the matrix that rotates a vector counterclockwise by

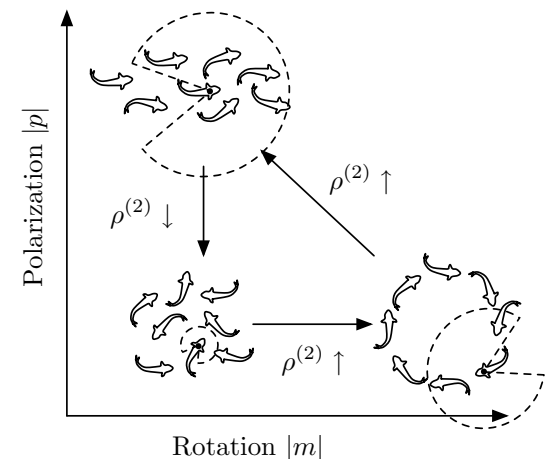

Fig. 1. Hysteresis loop in ROA behavior. Changing the radius $\rho^{(2)}$ of the zone of orientation (depicted by the dashed region centered on the dotted fish) generates transitions between swarm, circular, and parallel motion, which are characterized by collective polarization $|p|$ and rotation $|m|$.

an angle $\phi$ about the axis aligned with the vector $g_{k}(t+$ $\Delta t) \triangleq \hat{b}_{k}(t) \times \hat{f}_{k}(t+\Delta t)$. (If $\left|g_{k}(t+\Delta t)\right|=0$, then $R_{k}(t, \phi)$ is the identity matrix.) The deterministic version of ROA is $\hat{b}_{k}(t+\Delta t)=\left\{\begin{array}{c}\hat{f}_{k}(t+\Delta t), \quad\left|g_{k}(t+\Delta t)\right| \leq \sin \left(\omega_{0} \Delta t\right), \\ R_{k}\left(t, \omega_{0} \Delta t\right) \hat{b}_{k}(t), \quad \text { otherwise. }\end{array}\right.$

In the stochastic version, the deterministic behavior $\hat{b}_{k}(t+$ $\Delta t$ ) rotated by a small, random angle in a random direction.

The types of collective motion generated by the ROA behavior can be quantitatively distinguished using two metrics. Collective polarization measures the degree to which the particle velocities are aligned. The collective polarization vector $p(\hat{\mathbf{b}}(t)) \triangleq(1 / N) \sum_{j=1}^{N} \hat{b}_{j}(t)$ is proportional to the average linear momentum of the collective; the collective polarization is the magnitude $|p(\hat{\mathbf{b}}(t))|$ of the collective polarization vector. The second metric is proportional to the average angular momentum of the collective about the centroid $p_{r}(t) \triangleq(1 / N) \sum_{j=1}^{N} r_{j}(t)$ of the particle positions. This metric, called collective rotation, is the magnitude of the vector $m(\mathbf{r}(t), \hat{\mathbf{b}}(t)) \triangleq(1 / N) \sum_{j=1}^{N}\left(r_{j}(t)-p_{r}(t)\right) \times \hat{b}_{k}(t)$.

We use these metrics to characterize three different types of cohesive collective motion observed in numerical simulations of the ROA behavior illustrated in Figure 1: swarm motion, circular motion, and parallel motion. In cohesive collective motion, the collective does not fragment into smaller, isolated collectives. Swarm motion is characterized by low collective polarization and low collective rotation. Circular motion is characterized by low collective polarization and high collective rotation with individuals tending to be locally aligned with one another. Parallel motion is characterized by high collective polarization and low collective rotation.

The type of collective motion exhibited by the ROA behavior depends on the values of the model parameters and, in particular, the widths of the perceptual zones. Let $\Delta \rho_{k}^{(n)}=\Delta \rho_{j}^{(n)}$ for all pairs $j$ and $k$; in this case, we drop the subscripts $k$ and $j$. Extensive parametric studies using numerical investigations [4] have identified the width $\Delta \rho^{(2)}$ of the orientation zone and the width $\Delta \rho^{(3)}$ of the attraction zone as key parameters. For example, if $\Delta \rho^{(3)}$ is small, then 


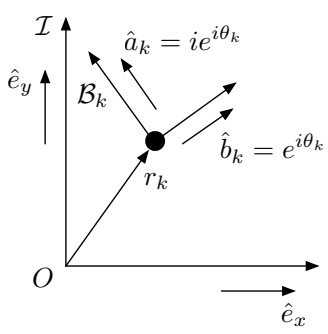

(a) Reference frames

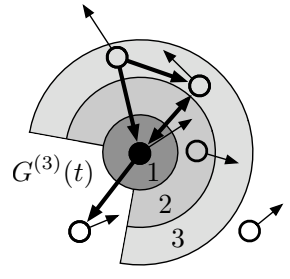

(b) Perceptual interaction
Fig. 2. Planar collective behavior model. (a) The direction of motion $\hat{b}_{k}=e^{i \theta_{k}}$ of particle $k$ lies in the span of the inertial reference frame unit vectors $\hat{e}_{x}$ and $\hat{e}_{y}$. (b) Three concentric, spherical perceptual zones with blind spot angles $\beta^{(0)}=0$ and $\beta^{2}=\beta^{3}=\pi / 4$; directed graph $G^{(3)}(t)$ generated by perceptual zones $\Gamma_{k}^{(3)}$ for all $k=1, \ldots, N$ is shown.

the collective tends to fragment, whereas, if $\Delta \rho^{(3)}$ is large, then the collective motion tends to be cohesive.

Transitions between three types of collective motion occur for large $\Delta \rho^{(3)}$ under slow variation of the width $\Delta \rho^{(2)}$ of the orientation zone [4]. If $\Delta \rho^{(2)}$ is small, then the collective moves in a swarm. For intermediate values of $\Delta \rho^{(2)}$, the collective exhibits either circular motion or parallel motion, whereas for large values of $\Delta \rho^{(2)}$, the collective exhibits parallel motion. If $\Delta \rho^{(2)}$ slowly increases, the collective transitions from swarm to circular to parallel motion; if $\Delta \rho^{(2)}$ slowly decreases, the collective transitions from parallel to circular to swarm motion. The value of $\Delta \rho^{(2)}$ at which the transition from circular to parallel motion occurs (for increasing $\Delta \rho^{(2)}$ ) is higher than the value at which the transition from parallel to circular motion occurs (for decreasing $\left.\Delta \rho^{(2)}\right)$. This hysteresis phenomenon, depicted in Figure 1, is called "collective memory" [4]: the type of collective motion depends on past values of model parameters that are not retained by individuals in the collective.

Collective memory occurs for large $\Delta \rho^{(3)}$ and intermediate values of $\Delta \rho^{(2)}$, since both circular and parallel motion are stable. We refer to the existence of two types of stable motion for the same parameter values as bistability (stability is used loosely here and in a Lyapunov sense below).

\section{SIMPLIFIED COLLECTIVE MODEL}

In order to find analytical evidence of bistability of circular and parallel motion in the collective behavior model, we simplify the model and isolate its fundamental components. The first simplification is to assume that the unit vector $\hat{e}_{z}$ is approximately parallel to the axis of rotation of the circular motion and project onto the plane spanned by the unit vectors $\hat{e}_{x}$ and $\hat{e}_{y}$; this yields a planar representation of the model.

To describe particle motion in a plane, assume that $\hat{b}_{k}(t) \times$ $\hat{a}_{k}(t)=\hat{e}_{z}$ as shown in Figure 2(a). We describe the direction of motion of particle $k$ by the phase $\theta_{k}(t) \in S^{1}$, that is, let $\hat{b}_{k}(t)=\cos \theta_{k}(t) \hat{e}_{x}+\sin \theta_{k}(t) \hat{e}_{y}$. To facilitate the planar analysis, we identify $\hat{e}_{x}$ and $\hat{e}_{y}$ with the complex numbers 1 and $i$, respectively. In complex notation, the velocity of particle $k$ is $\dot{r}_{k}(t)=s_{k}\left(\cos \theta_{k}(t)+i \sin \theta_{k}(t)\right)=s_{k} e^{i \theta_{k}(t)}$. The action of the steering behavior $\hat{b}_{k}(t+\Delta t)$ is to rotate the particle velocity $\dot{r}_{k}(t)$. Let $\boldsymbol{\theta}(t) \triangleq\left(\theta_{1}(t), \ldots, \theta_{N}(t)\right)^{T}$. Let $u_{k}(t+\Delta t)$ denote the angular rate of rotation, which is a function of the entries of $\mathbf{r}(t)$ and $\boldsymbol{\theta}(t)$ and the time step $\Delta t$. We have $\theta_{k}(t+\Delta t)=\theta_{k}(t)+u_{k}(t+\Delta t) \Delta t$.

The collective polarization vector $p(\hat{b}(t))$ is equivalent to $p_{\theta}(t) \triangleq(1 / N) \sum_{j=1}^{N} e^{i \theta_{j}}$, the phase order parameter [13], which satisfies $0 \leq\left|p_{\theta}(t)\right| \leq 1 ;\left|p_{\theta}(t)\right|=1$ if and only if $\theta_{j}=\theta_{k}$ for all pairs $j$ and $k$, i.e., phase synchronization. Phase synchronization corresponds to parallel motion. If all particles travel at the same speed, then $p_{\theta}(t)$ is proportional to the centroid velocity $\dot{p}_{r}(t)$. If $p_{\theta}(t)=0$, then the position centroid is fixed, which we call phase balancing.

To facilitate Lyapunov analysis, we express the collective behavior model as a system of ordinary differential equations. The discrete-time planar model is the forward Euler approximation of the continuous-time model given by

$$
\begin{aligned}
& \dot{r}_{k}=s_{k} e^{i \theta_{k}} \\
& \dot{\theta}_{k}=u_{k}(\boldsymbol{r}, \boldsymbol{\theta}) .
\end{aligned}
$$

We call (1) the particle model and $u_{k}$ the steering behavior. We do not assume that the particles have knowledge of the inertial frame $\mathcal{I}$ or any common reference frame. As a result, the steering behavior $u_{k}$ can depend only on the orientation and position of particle $j \in \mathcal{N}_{k}^{(n)}(t)$, for all $n$, relative to the orientation and position of particle $k$. We refer to the quantities $\theta_{k j}$ and $r_{k j} e^{-i \theta_{k}}$ as shape variables. Note $r_{k j} e^{-i \theta_{k}}$ is the position of particle $j$ relative to particle $k$ expressed as a vector component in frame $\mathcal{B}_{k}$. Provided the steering behavior $\boldsymbol{u} \triangleq\left(u_{1}, \ldots, u_{N}\right)^{T}$ depends only on shape variables and all particles travel at constant speed $s_{0}$, the particle model is invariant to rigid translation and rigid rotation of all of the particles, and both circular and parallel motion are relative equilibria [14].

Finally, to facilitate parametric studies, we nondimensionalize the particle model (1) using the inverse angular rate $\omega_{0}^{-1}$, which has units of time, and the characteristic length scale $\rho_{0} \triangleq s_{0} \omega_{0}^{-1}$, which has units of length; $\rho_{0}$ is the radius of the circle traced by a particle with $u_{k}=\omega_{0}$. We use the Fraktur typeface to represent dimensionless quantities: we have $\mathfrak{t} \triangleq t \omega_{0}, \mathfrak{r} \triangleq \boldsymbol{r} \rho_{0}^{-1}, \dot{\mathfrak{r}} \triangleq \dot{\boldsymbol{r}}\left(\rho_{0} w_{0}\right)^{-1}=\dot{\boldsymbol{r}} s_{0}^{-1}$, $\mathfrak{u} \triangleq \boldsymbol{u} \omega_{0}^{-1}$, and $\varrho^{(n)} \triangleq \rho^{(n)} \rho_{0}^{-1}$. Note that $\boldsymbol{\theta}$, which is in units of radians, is already dimensionless; we use $\dot{\boldsymbol{\theta}}$ to denote the dimensionless rate of change $d \boldsymbol{\theta} / d \mathfrak{t}$, which is a slight abuse of notation. The nondimensionalized particle model is $\dot{\mathfrak{r}}_{k}=e^{i \theta_{k}}$ and $\dot{\theta}_{k}=\mathfrak{u}_{k}(\mathfrak{r}, \boldsymbol{\theta})$. In this version of the particle model, a particle with $\mathfrak{u}_{k}=1$ travels around a unit circle.

The behavior rules are made concise using graph theory [9]. Let $G^{(n)}(t) \triangleq\left(\mathcal{N}, E^{(n)}(t)\right)$ denote a time-varying, directed graph that represents the interaction network generated by the $n$th perceptual zone, as shown in Figure 2(b). In graph $G^{(n)}(t)$, node $k$ corresponds to particle $k ; E^{(n)}$ is a set of ordered pairs $(j, k)$ that represent directed information flow from node $j$ to node $k$. Particle $j$ is contained in perceptual zone $\Gamma_{k}^{(n)}$ at time $t$ if and only if $(j, k) \in E^{(n)}(t)$. Assume that $E^{(n)}(t)$ is piecewise constant over finite time intervals. If there is a directed path in graph $G^{(n)}(t)$ from a node (the root) to every other node, then $G^{(n)}(t)$ is rooted. If 
$(j, k) \in E^{(n)}(t)$ if and only if $(k, j) \in E^{(n)}(t)$, then graph $G^{(n)}(t)$ is undirected. Note, $G^{(n)}(t)$ is undirected if all of the particles have omnidirectional perception with respect to perceptual zone $n$. An undirected graph is connected if and only if there is a path between every pair nodes.

The Laplacian matrix of a graph can be used to approximate the ROA behavior rules. The Laplacian matrix $L^{(n)}=\left[l_{k j}^{(n)}(t)\right]$ of graph $G^{(n)}(t)$ is an $N \times N$ matrix. The off-diagonal entries of the Laplacian are $l_{k j}^{(n)}(t)=-1$, if $j \in \mathcal{N}_{k}^{(n)}(t)$, otherwise $l_{k j}^{(n)}(t)=0$. The diagonal entries of the Laplacian are $l_{k k}^{(n)}(t) \triangleq\left|\mathcal{N}_{k}^{(n)}(t)\right|$. Let $d_{k}^{(n)}(t) \triangleq$ $\max \left\{\left|\mathcal{N}_{k}^{(n)}(t)\right|, 1\right\}$ and $\boldsymbol{d}^{(n)}(t) \triangleq\left(d_{1}^{(n)}(t), \ldots, d_{N}^{(n)}(t)\right)^{T}$. The normalized Laplacian $\tilde{L}^{(n)}(t) \triangleq\left[\tilde{l}_{k j}^{(n)}(t)\right]$ has components $\tilde{l}_{k j}^{(n)}(t)=l_{k j}^{(n)}(t) / d_{k}^{(n)}(t)$ for all pairs $j$ and $k$. Let $\tilde{L}_{k}^{(n)}(t)$ denote its $k$ th row. We have

$$
\tilde{L}_{k}^{(n)} \boldsymbol{r}=r_{k}-\frac{1}{d_{k}^{(n)}(t)} \sum_{j \in \mathcal{N}_{k}^{(n)}(t)} r_{j},
$$

which is a vector from the position centroid of the particles in perceptual zone $\Gamma_{k}^{(n)}$ to position $r_{k}$. Let $\langle x, y\rangle$ denote the real inner product of two complex numbers $x$ and $y$, that is, $\langle x, y\rangle \triangleq \operatorname{Re}\left\{x^{*} y\right\}$, where $x^{*}$ is the complex conjugate of $x$.

Let $K_{0}$ be any real number not equal to zero. Consider

$$
u_{k}=K_{0}\left\langle i e^{i \theta_{k}}, \tilde{L}_{k}^{(n)} \boldsymbol{r}\right\rangle .
$$

If $\left|\mathcal{N}_{k}^{(n)}(t)\right|=0$ or $\tilde{L}_{k}^{(n)} \boldsymbol{r}=0$, then $\dot{\theta}_{k}=u_{k}=0$. The rule (3) is also zero for motion of particle $k$ toward or away from the position centroid of the particles in zone $\Gamma_{k}^{(n)}$; these are equilibrium states of $\dot{\theta}_{k}=u_{k}$. For nonequilibrium states, if $K_{0}>0$ and $n=1$, then $u_{k}$ stabilizes (resp. destabilizes) motion of particle $k$ away from (resp. toward) the position centroid of particles in the repulsion zone $\Gamma_{k}^{(1)}$. This is because if $\theta_{k}>\arg \left\{\tilde{L}_{k}^{(n)} \boldsymbol{r}\right\}$, then $\dot{\theta}_{k}<0$ and, if $\theta_{k}<\arg \left\{\tilde{L}_{k}^{(n)} \boldsymbol{r}\right\}$, then $\dot{\theta}_{k}>0$. By this reasoning, we conclude (3) with $K_{0}>0$ is a repulsion behavior rule. By similar reasoning, if $K_{0}<0$ and $n=3$, then $u_{k}$ stabilizes (resp. destabilizes) motion toward (resp. away from) the position centroid of particles in the attraction zone $\Gamma_{k}^{(3)}(t)$, which implies (3) with $K_{0}<0$ is an attraction behavior rule.

We approximate the orientation behavior rule in a similar way. Let $e^{i \boldsymbol{\theta}} \triangleq\left(e^{i \theta_{1}}, \ldots, e^{i \theta_{N}}\right)^{T}$. Consider the behavior rule

$$
u_{k}=-\left\langle i e^{i \theta_{k}}, \tilde{L}_{k}^{(n)} e^{i \boldsymbol{\theta}}\right\rangle=\left\langle i e^{i \theta_{k}}, \frac{1}{d_{k}^{(n)}} \sum_{j \in \mathcal{N}_{k}^{(n)}} e^{i \theta_{j}}\right\rangle .
$$

Note that the term $\left(1 / d_{k}^{(n)}\right) \sum_{j \in \mathcal{N}_{k}^{(n)}} e^{i \theta_{j}} \triangleq p_{k, \theta}^{(n)}$ represents the polarization vector of the particles in zone $\Gamma_{k}^{(n)}$. Let $n=2$. If $\left|\mathcal{N}_{k}^{(2)}\right|=0$ or $\tilde{L}_{k}^{(2)} e^{i \boldsymbol{\theta}}=0$, then $u_{k}=0$. The rule (4) is also zero for motion of particle $k$ parallel or antiparallel to the average direction of motion of the particles in zone $\Gamma_{k}^{(n)}$, which are equilibrium states of $\dot{\theta}_{k}=u_{k}$. For non-equilibrium states, if $\theta_{k}>\arg \left\{p_{k, \theta}^{(2)}\right\}$, then $\dot{\theta}_{k}<0$ and, if $\theta_{k}<\arg \left\{p_{k, \theta}^{(2)}(t)\right\}$, then $\dot{\theta}_{k}>0$. This implies that the behavior rule (4) stabilizes (resp. destabilizes) motion of particle $k$ parallel (resp. anti-parallel) to the average direction of motion of the particles in its attraction zone.

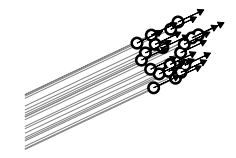

(a) $\varrho^{(1)}=0.1, K_{0}=1$

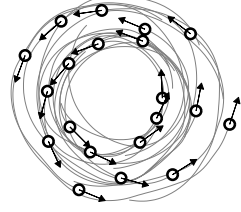

(b) $\varrho^{(1)}=0.5, K_{0}=0.1$
Fig. 3. Bistability of circular and parallel motion of the particle model (1) with the omnidirectional control (11). Parameters values: $N=20, \rho_{0}=10$, $\varrho^{(2)}=1$, and $\varrho^{(3)}=2$. (a) Initial positions chosen randomly from a uniform distribution on a $\rho_{0} \times \rho_{0}$ domain, initial phases from $\left[-\frac{\pi}{5}, \frac{\pi}{5}\right] \bmod$ $2 \pi$; (c) initial positions from $2 \rho_{0} \times 2 \rho_{0}$, initial phases from $[0,2 \pi)$.

Let $\operatorname{sat}(x)$ denote the saturation function, where sat $(x)=$ $x$, if $|x| \leq 1$, and $\operatorname{sat}(x)=\operatorname{sgn}(x)$, if $|x|>1$. We use this notation to approximate the deterministic version of the ROA behavior in two dimensions: $u_{k}=\omega_{0} \operatorname{sat}\left(\left\langle i e^{i \theta_{k}},\left(\tilde{L}_{k}^{(1)}-\right.\right.\right.$ $\left.\left.\left.\tilde{L}_{k}^{(3)}\right) \boldsymbol{r}-\tilde{L}_{k}^{(2)} e^{i \boldsymbol{\theta}}\right\rangle\right)$. The nondimensionalized form is

$$
\mathfrak{u}_{k}=\operatorname{sat}\left(\left\langle i e^{i \theta_{k}},\left(\tilde{L}_{k}^{(1)}-\tilde{L}_{k}^{(3)}\right) \mathfrak{r}-\tilde{L}_{k}^{(2)} e^{i \boldsymbol{\theta}}\right\rangle\right) .
$$

For $\left|f\left(x_{0}\right)\right| \leq 1$, the first-order Taylor series approximation of sat $(f(x))$ about $x_{0}$ is $f\left(x_{0}\right)+(d f / d x)\left(x-x_{0}\right)$. Therefore, linearization of (5) generates a constant term $f\left(x_{0}\right)$, which is zero for parallel motion and nonzero for circular motion.

For the steering behavior (5), numerical simulations of (1) indeed reveal the co-existence of different types of cohesive collective motion in various parameter ranges. In particular, we simulated a characteristic length scale $\rho_{0}=$ 10 , blind spot angle $\beta_{k}=\pi / 4$ for all $k$, and repulsion and attraction zone dimensionless radii $\varrho^{(1)}=0.1$ and $\varrho^{(3)}=5$, respectively. Starting from initial positions chosen randomly from a uniform distribution in a $2 \times 2$ domain and initial phases chosen randomly from a uniform distribution in $[0,2 \pi)$, we observe that swarm motion occurs in the range $0 \leq \varrho^{(2)} \leq 0.5$, circular motion occurs in the range $0.3 \leq \varrho^{(\overline{2})} \leq 0 . \overline{7}$, and parallel motion occurs in the range $\varrho^{(2)} \geq 0.5$. This suggests that circular motion arises among locally aligned individuals, whereas parallel motion occurs when individuals are globally aligned. Saturating the angular rate appears important to achieve circular motion; for the steering behavior (5), particles traveling in circular motion are turning at nearly the maximum rate.

For the steering behavior (5) and the parameter range for which circular motion occurs, there exists a set of initial conditions for which parallel motion results. For example, consider the set of initial conditions for which $\theta_{j}(0)=\theta_{k}(0)$ for all pairs $j$ and $k$. Assume the perceptual zone radii are in a range that supports circular motion. Let $E^{(1)}(0)=\emptyset$ and $E^{(3)}(0)=\emptyset$. If the graph $G_{k}^{(2)}(t)=G_{k}^{(2)}(0)$ is undirected and connected, then, by Proposition 1 below, the collective exhibits parallel motion. Although this example is trivial, it shows that the steering behavior (5) is bistable.

\section{OMNIDIRECTIONAL PERCEPTION}

In this section, we describe analytical evidence for bistability of circular and parallel motion in a steering behavior 
that, like the ROA behavior, contains a linear combination of behavior rules. The analysis, which is based on Lyapunov stability theory, applies to collectives with omnidirectional perception. Recall that omnidirectional perception generates interaction networks that can be described by undirected graphs. The Laplacian matrix of an undirected graph is, by definition, symmetric, which leads to the definition of the Laplacian quadratic form. We describe below two Laplacian quadratic forms that serve as Lyapunov functions.

Let $L^{(n)}$ be the Laplacian of a time-invariant, undirected graph $G^{(n)}=\left(\mathcal{N}, E^{(n)}\right)$. Let $\langle\boldsymbol{x}, \boldsymbol{y}\rangle \triangleq \sum_{j=1}^{N}\left\langle x_{j}, y_{j}\right\rangle$, where $\boldsymbol{x}$ and $\boldsymbol{y}$ are $N \times 1$ complex matrices. A Laplacian quadratic form is $Q_{L^{(n)}}(\boldsymbol{x}) \triangleq\left\langle\boldsymbol{x}, L^{(n)} \boldsymbol{x}\right\rangle$. Let $\mathbf{1} \triangleq(1, \ldots, 1)^{T}$ be a $N \times 1$ matrix of ones. If $G^{(n)}$ is connected, then $Q_{L^{(n)}}(\boldsymbol{x}) \geq$ 0 and $Q_{L^{(n)}}(\boldsymbol{x})=0$ if and only if $\boldsymbol{x} \in \operatorname{span}\{\mathbf{1}\}$ [15].

We use the Laplacian quadratic form $Q_{L}(\boldsymbol{x})$ to define the Laplacian phase potential $W_{L^{(n)}}(\boldsymbol{\theta}) \triangleq(1 / 2) Q_{L^{(n)}}\left(e^{i \boldsymbol{\theta}}\right)=$ $(1 / 2)\left\langle e^{i \boldsymbol{\theta}}, L^{(n)} e^{i \boldsymbol{\theta}}\right\rangle$ [8]. The gradient of $W_{L^{(n)}}(\boldsymbol{\theta})$ is $\frac{\partial W_{L}(n)}{\partial \boldsymbol{\theta}} \triangleq\left(\frac{\partial W_{L}(n)}{\partial \theta_{1}}, \ldots, \frac{\partial W_{L}(n)}{\partial \theta_{N}}\right)^{T}$, where, using $L^{(n)}=$ $\left(L^{(n)}\right)^{T}$, we have $\frac{\partial W_{L}(n)}{\partial \theta_{k}}=\left\langle i e^{i \theta_{k}}, L_{k}^{(n)} e^{i \boldsymbol{\theta}}\right\rangle$. Note, the $k$ th entry in the gradient of the Laplacian phase potential with $n=2$ is proportional to the orientation behavior rule (4). This leads to the following result, based on [8, Section III.B].

Proposition 1: Let $L^{(2)}(t)$ be the Laplacian of the undirected graph $G^{(2)}(t)=\left(\mathcal{N}, E^{(2)}(t)\right)$ that describes the orientation zone interaction network. For the steering behavior (4) with $n=2$, consider the set $\Lambda$ of solutions of (1) where $G^{(2)}(t)$ is time-invariant and connected. All of the solutions in $\Lambda$ with a synchronized phase arrangement are asymptotically stable.

Proof: The time-derivative of the Laplacian phase potential $W_{L^{(2)}}(\boldsymbol{\theta})$ along solutions of (1) satisfies $\dot{W}_{L^{(2)}} \leq 0$. By the invariance principle, all of the solutions converge to the largest invariant set for which $\left\langle i e^{i \theta_{k}}, L_{k}^{(2)} e^{i \boldsymbol{\theta}}\right\rangle=0$, which contains the set of synchronized phase arrangements. The synchronized set is asymptotically stable because it is the global minimum of $W_{L^{(2)}}(\boldsymbol{\theta})$.

Proposition 1 is limited because it applies only to timeinvariant and undirected interaction networks. Nonetheless, it provides analytic evidence to support the observations of numerical simulations of the orientation behavior rule (4). Next, we provide evidence to show that a variation of the attraction/repulsion behavior (3) stabilizes circular motion.

A variation of behavior (3) is

$$
u_{k}=K_{0}\left\langle e^{i \theta_{k}}, \tilde{L}_{k}^{(n)} \boldsymbol{r}\right\rangle .
$$

If $\left|\mathcal{N}_{k}^{(n)}(t)\right|=0$ or $\tilde{L}_{k}^{(n)} \boldsymbol{r}=0$, then $\dot{\theta}_{k}=u_{k}=0$. The rule (6) is also zero for motion of particle $k$ perpendicular to $\tilde{L}_{k}^{(n)} \boldsymbol{r}$; these are equilibrium states of $\dot{\theta}_{k}=u_{k}$. For nonequilibrium states, if $\theta_{k}>\arg \left\{\tilde{L}_{k}^{(n)} \boldsymbol{r}\right\}+(\pi / 2) \operatorname{sgn}\left(K_{0}\right)$, then $\dot{\theta}_{k}<0$ and, if $\theta_{k}<\arg \left\{\tilde{L}_{k}^{(n)} \boldsymbol{r}\right\}+(\pi / 2) \operatorname{sgn}\left(K_{0}\right)$, then $\dot{\theta}_{k}>0$. By this reasoning, we conclude the behavior rule (6) stabilizes revolute motion of particle $k$ around the position centroid of particles in zone $\Gamma_{k}^{(n)}$; we call (6) a revolution behavior rule. The direction of revolution depends on the sign of $K_{0}$.
Consider collective circular motion about a fixed point. Assume the radius of the circle is $s_{0}\left|\omega_{0}\right|^{-1}$ and the direction of rotation is determined by $\operatorname{sgn}\left(\omega_{0}\right)$. Let $c_{k}$ denote the center of the circle orbited by particle $k$, that is,

$$
c_{k}(t) \triangleq r_{k}(t)+s_{0} \omega_{0}^{-1} i e^{i \theta_{k}(t)} .
$$

Let $\boldsymbol{c}(t) \triangleq\left(c_{1}(t), \ldots, c_{N}(t)\right)^{T}$. Let $L^{(n)}$ be the Laplacian of a time-invariant, undirected graph $G^{(n)}=\left(\mathcal{N}, E^{(n)}\right)$. A Laplacian quadratic form associated to the circular motion is $S_{L^{(n)}}(\boldsymbol{r}, \boldsymbol{\theta}) \triangleq(1 / 2) Q_{L^{(n)}}(\boldsymbol{c})=(1 / 2)\left\langle\boldsymbol{c}, L^{(n)} \boldsymbol{c}\right\rangle$, which we call the Laplacian circle potential. If $G^{(n)}$ is connected, then $S_{L}(\boldsymbol{r}, \boldsymbol{\theta}) \geq 0$ and $S_{L}(\boldsymbol{r}, \boldsymbol{\theta})=0$ if and only if $c_{k}=c_{j}$ for all pairs $j$ and $k$. This leads to the following result, which is based on [8, Theorem 5].

Proposition 2: Let $L^{(3)}(t)$ be the Laplacian of the undirected graph $G^{(3)}(t)=\left(\mathcal{N}, E^{(3)}(t)\right)$ that describes the attraction zone interaction network. For the steering behavior

$$
u_{k}=\omega_{0}\left(1+K_{0}\left\langle e^{i \theta_{k}}, \tilde{L}_{k}^{(3)} \boldsymbol{c}\right\rangle\right), K_{0}>0,
$$

consider the set $\Lambda$ of solutions of (1) where $G^{(3)}(t)$ is timeinvariant and connected. All of the solutions in $\Lambda$ asymptotically converge to circular motion with radius $s_{0}\left|\omega_{0}\right|^{-1}$ and direction of rotation determined by $\operatorname{sgn}\left(\omega_{0}\right)$.

Proof: The time-derivative of the Laplacian circle potential $S_{L^{(3)}}(\boldsymbol{\theta})$ along solutions of (1) satisfies $\dot{S}_{L^{(3)}} \leq 0$. By the invariance principle, all of the solutions converge to the largest invariant set for which $\left\langle e^{i \theta_{j}}, L_{j}^{(2)} c\right\rangle=0$. In this set, $\dot{\boldsymbol{\theta}}=\omega_{0} \mathbf{1}$ and $c_{k}=c_{j}$ for all pairs $j$ and $k$.

It can be shown that a linear combination of the steering behaviors (4) with $n=3$ and (8) stabilizes circular motion in which all of the phases are synchronized. In fact, using (7), the circle steering behavior (8) can be written $u_{k}=$ $\omega_{0}\left(1+K_{0}\left\langle e^{i \theta_{k}}, \tilde{L}_{k}^{(3)} \boldsymbol{r}\right\rangle\right)-s_{0} K_{0}\left\langle i e^{i \theta_{k}}, \tilde{L}_{k}^{(3)} e^{i \boldsymbol{\theta}}\right\rangle$, which is a linear combination of a constant term $\omega_{0}$, the revolution behavior rule (6), and the orientation behavior rule (4). Let $H(x)$ denote the Heaviside step function, where $H(x)=0$ if $x<0, H(x)=1 / 2$ if $x=0$, and $H(x)=1$ if $x>0$.

Theorem 1: Let $L^{(n)}(t)$ be the Laplacian of the undirected graph $G^{(n)}(t)=\left(\mathcal{N}, E^{(n)}(t)\right)$ that describes the interaction network for zone $n \in\{1,2,3\}$. For the steering behavior

$$
\begin{aligned}
u_{k}= & \omega_{0}\left(H\left(\left|\mathcal{N}_{k}^{(3)}\right|\right)+K_{0}\left\langle e^{i \theta_{k}}, \tilde{L}_{k}^{(3)} \boldsymbol{r}\right\rangle\right)- \\
& s_{0} K_{0}\left\langle i e^{i \theta_{k}}, \tilde{L}_{k}^{(2)} e^{i \boldsymbol{\theta}}-\tilde{L}_{k}^{(1)} \boldsymbol{r}\right\rangle, K_{0}>0,
\end{aligned}
$$

consider the set $\Lambda$ of solutions of (1) where $G^{(n)}(t)=$ $G^{(n)}(0)$ for all $n$. All of the solutions in $\Lambda$ for which $G^{(2)}$ is connected, $E^{(1)}=E^{(3)}=\emptyset$, and the phase arrangement is synchronized are asymptotically stable. All of the solutions in $\Lambda$ for which $G^{(3)}$ is connected and $E^{(1)}=E^{(2)}=$ $\emptyset$ asymptotically converge to circular motion with radius $s_{0}\left|\omega_{0}\right|^{-1}$ and direction of rotation determined by $\operatorname{sgn}\left(\omega_{0}\right)$.

Proof: In the case $G^{(2)}$ is connected and $E^{(1)}=E^{(3)}=$ $\emptyset$, then the proof follows from Proposition 1. Assuming $G^{(3)}$ is connected and $E^{(1)}=E^{(2)}=\emptyset$, then (9) is equivalent to

$$
\begin{aligned}
u_{k}= & \omega_{0}\left(1+K_{0}\left\langle e^{i \theta_{k}}, \tilde{L}_{k}^{(3)} \boldsymbol{r}\right\rangle\right)+ \\
& \left(K-s_{0} K_{0}\right)\left\langle i e^{i \theta_{k}}, \tilde{L}_{k}^{(3)} e^{i \boldsymbol{\theta}}\right\rangle \\
= & \omega_{0}\left(1+K_{0}\left\langle e^{i \theta_{k}}, \tilde{L}_{k}^{(3)} \boldsymbol{c}\right\rangle\right)+K\left\langle i e^{i \theta_{k}}, \tilde{L}_{k}^{(3)} e^{i \boldsymbol{\theta}}\right\rangle
\end{aligned}
$$



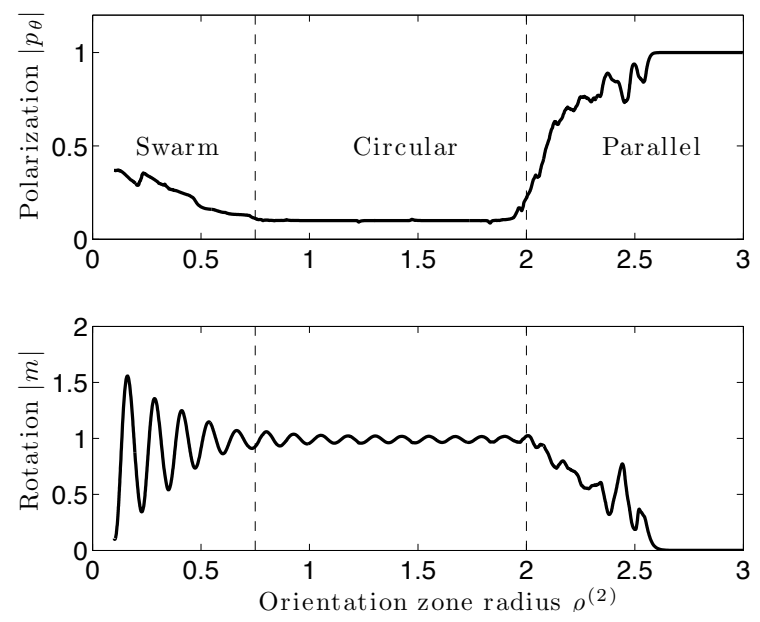

Fig. 4. For the behavior (11) with $\rho_{0}=10, \varrho^{(1)}=0.1, \varrho^{(3)}=0.4$, $K_{0}=0.1$ and random initial conditions, increasing the orientation zone radius $\varrho^{(2)}$ generates transitions from swarm to circular to parallel motion.

where $K=s_{0} K_{0}>0$. For this steering behavior, the timederivative of the composite Laplacian potential $V_{L^{(3)}}(\boldsymbol{r}, \boldsymbol{\theta})=$ $\omega_{0}^{2} S_{L^{(3)}}(\boldsymbol{r}, \boldsymbol{\theta})-K W_{L^{(3)}}(\boldsymbol{\theta})$ satisfies $\dot{V}_{L^{(3)}} \leq 0$. By the invariance principle, all of the solutions converge to the largest invariant set $\Omega$ for which

$$
\omega_{0} K_{0}\left\langle e^{i \theta_{j}}, L_{j}^{(3)} \boldsymbol{c}\right\rangle+K\left\langle i e^{i \theta_{j}}, L_{j}^{(3)} e^{i \boldsymbol{\theta}}\right\rangle=0 .
$$

By taking the time-derivative of (10) in $\Omega$, one can show that $\dot{\boldsymbol{\theta}}=\omega_{0} \mathbf{1}$ and $c_{k}=c_{j}$ for all pairs $j$ and $k$.

Theorem 1 shows that the steering behavior (9) stabilizes both parallel and circular motion for the same parameter values. Nondimensionalizing (9) yields

$$
\begin{aligned}
& \mathfrak{u}_{k}= \pm\left(H\left(\left|\mathcal{N}_{k}^{(3)}\right|\right)+K_{0}\left\langle e^{i \theta_{k}}, \tilde{L}_{k}^{(3)} \mathfrak{r}\right\rangle\right)- \\
& \rho_{0} K_{0}\left\langle i e^{i \theta_{k}}, \tilde{L}_{k}^{(2)} e^{i \boldsymbol{\theta}}-L_{k}^{(1)} \mathfrak{r}\right\rangle,
\end{aligned}
$$

where the positive (resp. negative) sign corresponds to counterclockwise (resp. clockwise) rotation. In the simulation shown in Figure 3(a), we used $\varrho^{(1)}=0.1$ and $\varrho^{(3)}=5$. For these values, steering behavior (11) with $\varrho^{(2)}=1$ generates either circular or parallel motion, depending on the initial conditions. Increasing $\varrho^{(2)}$ tends to yield parallel motion only, whereas decreasing $\varrho^{(2)}$ tends to yield only circular motion; these tendencies are generally consistent with observations of the ROA behavior. For these parameter values, the circular motion is characterized by two opposing clusters of particles. Increasing $\varrho^{(1)}$ and/or decreasing $\varrho^{(2)}$ more evenly distributes the particle formation Figure 3(b).

Theorem 1 implies that the particle model may exhibit hysteresis under slow variation of the radius of the zone of orientation, although simulation results are inconclusive. Figure 4 shows simulation results of the nondimensionalized particle model with the control (11). Starting from random initial conditions, slowly increasing the radius $\varrho^{(2)}$ of the orientation zone from 0.1 to 3.0 generates transitions from swarm to circular to parallel; starting from parallel motion, slowly decreasing $\varrho^{(2)}$ causes fragmentation.
The repulsion behavior rule, which only activates if $\left|\mathcal{N}_{k}^{(1)}(t)\right|>0$, steers particle $k$ away from collisions. However, unlike the ROA behavior, the direction of rotation of the circular motion generated by (9) is not random, even for random initial conditions. This limitation has not yet been addressed. Another limitation of (9) that has been addressed (but omitted for space constraints) is the restriction to timeinvariant and undirected interaction networks. Lifting this restriction comes at the cost of increased complexity.

\section{CONCLUSIONS}

This paper describes idealized yet understandable models of collective motion that are bistable with respect to circular and parallel motion. The primary contribution is to support numerical simulation results of a collective behavior model with analytical results for a related model, using tools from cooperative control theory. The latter model and the corresponding steering behaviors suffer overall from lack of biological plausibility. However, the seemingly artificial use of the Heaviside step function to "activate" the constant turning rate in (9) when neighboring particles are perceived, may be related to quorum sensing in bacteria [16].

\section{REFERENCES}

[1] H. Levine, E. Ben-Jacob, I. Cohen, and W.-J. Rappel, "Swarming patterns in microogranisms: Some new modeling results," in Proc. IEEE CDC, 2006, pp. 5073-5077.

[2] J. Buhl, D. J. T. Sumpter, I. D. Couzin, J. J. Hale, E. Despland, E. R. Miller, and S. J. Simpson, "From disorder to order in marching locusts," Science, vol. 312, no. 5778, pp. 1402-1406, 2006.

[3] J. K. Parrish, S. V. Viscido, and D. Grünbaum, "Self-organized fish schools: An examination of emergent properties," Biol. Bull., vol. 202, no. 3, pp. 296-305, 2002.

[4] I. D. Couzin, J. Krause, R. James, G. D. Ruxton, and N. R. Franks, "Collective memory and spatial sorting in animal groups," J. Theor Biol., vol. 218, no. 1, pp. 1-11, 2002.

[5] A. Huth and C. Wissel, "The movement of fish schools: A simulation model," in Biological Motion, Lec. Notes in Biomathematics, W. Alt and G. Hoffmann, Eds. Springer-Verlag, 1990, no. 89, pp. 577-590.

[6] D. Paley, N. E. Leonard, and R. Sepulchre, "Collective motion: Bistability and trajectory tracking," in Proc. IEEE CDC, 2004, pp. 1932-1937.

[7] R. Sepulchre, D. A. Paley, and N. E. Leonard, "Stabilization of planar collective motion: All-to-all communication," IEEE Trans. Automatic Control, vol. 52, no. 5, pp. 811-824, 2007.

[8] —, "Stabilization of planar collective motion with limited communication," IEEE Trans. Automatic Control, accepted.

[9] C. Godsil and G. Royle, Algebraic Graph Theory, ser. Graduate Texts in Mathematics. Springer-Verlag, 2001, no. 207.

[10] W. Ren and R. W. Beard, "Consensus seeking in multiagent systems under dynamically changing interaction topologies," IEEE Trans. Automatic Control, vol. 50, no. 5, pp. 655-661, 2005.

[11] D. A. Paley, N. E. Leonard, and R. Sepulchre, "Collective motion of self-propelled particles: Stabilizing symmetric formations on closed curves," in Proc. IEEE CDC, 2006, pp. 5067-5072.

[12] E. W. Justh and P. S. Krishnaprasad, "Natural frames and interacting particles in three dimensions," in Proc. Joint IEEE CDC/ECC, 2005 , pp. 2841-2846.

[13] Y. Kuramoto, "Self-entrainment of a population of coupled non-linear oscillators," in Proc. Int. Symp. Mathematical Problems in Theoretical Physics. Kyoto, Japan: Kyoto University, January 1975, pp. 420-422.

[14] E. W. Justh and P. S. Krishnaprasad, "Equilibria and steering laws for planar formations," Systems \& Control Letters, vol. 52, no. 1, pp. $25-38,2004$.

[15] R. Merris, "Laplacian matrices of graphs: A survey," Linear Algebra and its Applications, vol. 197, no. 198, pp. 143-176, 1994.

[16] M. B. Miller and B. L. Bassler, "Quorum sensing in bacteria," Аnпи. Rev. Microbiol., vol. 55, pp. 165-199, October 2001. 